Journal of Animal and Veterinary Advances 11 (15): 2641-2646, 2012

ISSN: $1680-5593$

(C) Medwell Journals, 2012

\title{
Cryotherapy Combined with Warm Transfusion Apply to Serious Burns in a Rabbit Model: An Experimental Study
}

\author{
Chunliu Lv, Pihong Zhang, Xiaoyuan Huang, Jizhang Zeng, Licheng Ren and Pengfei Liang \\ Department of Burn and Reconstructive Surgery, Xiangya Hospital, \\ Central South University, 410008 Changsha, China
}

\begin{abstract}
This study investigated the effect of cryotherapy combined with warm transfusion on vital signs, inflammatory response and tissues permeability in rabbits with severe burns. Rabbits were subjected to deep partial-thickness scald covering of $40 \%$ Total Body Surface Area (TBSA). Rabbits were randomly divided into 4 groups namely scald group (A), cryotherapy after scald group (B), warm transfusion after scald group (C), group of cryotherapy combined with warm transfusion after scald (D). Cryotherapy meant soaking the burn wound in $10^{\circ} \mathrm{C}$ cold water for $40 \mathrm{~min}$ after scald $5 \mathrm{~min}$. Fluid of warm transfusion was maintained at 39 . This data shown that cryotherapy decreased the core body temperature, Mean Arterial Pressure (MAP) and wound Myeloperoxidase (MPO) activity in rabbits and increased the levels of reduced shear viscosity/erythrocyte aggregation and inflammatory mediators compared to control group. The upper indicators produced the opposite changes under warm transfusion. Moisture content of lung in group $\mathrm{C}$ and moisture content of wound in group $\mathrm{B}$ were lower compared to group $\mathrm{A}$. Moreover, burn wound $\mathrm{MPO}$, moisture content of lung and wound and inflammatory mediators significantly descended in group D.
\end{abstract}

Key words: Burns, cryotherapy, core body temperature, warm transfusion, rabbits

\section{INTRODUCTION}

Cryotherapy means washing or soaking the wounds with cold water and is universally acknowledged as an effective way to treat burn patients during emergencies. Evidence verifies that immediate cooling of animals with full thickness burns will reduce mortality by allievating tissue damage and promoting wound healing (Ofeigsson et al., 1972; Meusers et al., 1972; Jandera et al., 2000). Cryotherapy is effective at removing noxious agents and alleviating pain. It may also reduce edema by stabilizing mast cells and histamine release (Jackie and Sukh, 2004) even improve graft survival (Hirase, 1993). Despite all these advantages of cryotherapy, controversy still exists concerning the scope of its application. Large area cryotherapy can lead to hypothermia and can even provoke or exacerbate shock. So, cryotherapy is rarely applied to large burn patients. Hypothermia is also considered as an independent risk factor of trauma mortality (Shafi et al., 2005). Excessive and prolonged cooling will deepen burn wounds due to local vasoconstriction or blood circulation disorder and cause severe proliferative scar after healing (Sawada et al., 1997). Experimental studies show that radiant furnace is used for heat-preservation of severe burn patients at the same time as cooling and this method achieves preferable therapeutic effects (Schlager and Iserson, 1988). So far, insufficient attention has been focused on how to prevent the side effects of cryotherapy. Warm transfusion which beginning to be used from the last century has become popularized for significantly shortening the duration of low body temperature. Intravenous fluid warming is an important method of heat conservation. Maintaining normothermia intraoperatively is likely to decrease the incidence of infectious complications in patients undergoing colorectal resection and to shorten their hospitalizations (Kurz et al., 1996). Clinically, a warmer should be used if the body temperature from fluid administration is likely to decrease $>0.5-1.0^{\circ} \mathrm{C}$ (Horowitz et al., 2004). During operation, hemodynamic situation (core and skin temperatures, MAP), shivering and recovery time is less in the group that receiving warming intravenous fluid than the group that receiving fluids at room temperature (Hasankhani et al., 2007). Research shows that warm transfusion in large burn patients during shock phase could significantly improve the effect of fluid resuscitation.

Therefore, researchers developed a new method of cryotherapy combined with warm transfusion applying to

Corresponding Author: Pihong Zhang, Department of Burn and Reconstructive Surgery, Xiangya Hospital,

Central South University, 410008 Changsha, China 
rabbits with severe burns. The aim of this pilot study was to evaluate the efficacy of this method in preventing hypothermia and severe shock potentially caused by cryotherapy and discussed the feasibility and superiority of cryotherapy combined with warm transfusion used in early treatments of large burn wound.

\section{MATERIALS AND METHODS}

Animal: The experiment was performed in accordance with the Animal Care Guidelines and approved by the animal research ethics committee of Central South University. Healthy adult rabbits (Laboratory Animal Department of Central South University) either male or female weighing from $1.5-2.0 \mathrm{~kg}$ were used. The animals were subjected to fasting for $12 \mathrm{~h}$ before scald but had free access to water. The rabbits were randomly divided into 4 groups of 6 each namely scald group (group A), cryotherapy after scald group (group B), warm transfusion after scald group (group C) and group of cryotherapy combined with warm transfusion after scald (group D).

Reagents and instruments: MPO kit and ELISA kits of TNF $\alpha$, IL-1 $\beta$, IL- 6 were purchased, respectively form Nan Jing Jian cheng Biotechnology Institute (China) and the ADL company (USA). ECG (Philips), Warm Transfusion Device (Steve Lang) and Microplate Reader (Heraeus) were also used.

Experimental protocol: The injury procedures were under sodium pentobarbital anesthesia $\left(25 \mathrm{mg} \mathrm{kg}^{-1} \mathrm{IV}\right)$. Rabbits were subjected to deep partial-thickness scald covering of $40 \%$ TBSA by immersing to hot water $\left(95^{\circ} \mathrm{C}\right)$ for $15 \mathrm{sec}$ confirmed by pathological study. The cryotherapy meant soaking the $40 \%$ TBSA wound in $10^{\circ} \mathrm{C}$ cold water for $40 \mathrm{~min}$ after scald $5 \mathrm{~min}$ (Li et al., 1997). Ringer's lactate solution was transfused in each group for $48 \mathrm{~h}$ by parkland formula via marginal ear vein after injury, the fluid of warm transfusion was maintained at $39^{\circ} \mathrm{C}$ with warming device and the normal transfusion was at about $23^{\circ} \mathrm{C}$ (Nakazawa and Nozaki, 2005). MAP was obtained by exposing the carotid artery and inserting a catheter which connected with ECG. Core body temperature was measured by the thermistor positioned at the rectum. All samples were phlebotomized at 2, 24 and $48 \mathrm{~h}$ after injury then plasma inflammatory mediators (TNF $\alpha, \mathbb{L}-1 \beta, \mathrm{IL}-6)$ and hemorheological parameters (Reduced low shear viscosity (Rls), Reduced high shear viscosity (Rhs), Plasma Viscosity (PV), Erythrocyte Aggregation (EA)) were measured. Burn wound tissues were taken at 2 , 24 and $48 \mathrm{~h}$ after injury and tested for MPO activity. Tissues from the wound and lung were also taken at $48 \mathrm{~h}$ after injury and moisture contents were calculated according to the following equation:
Water content $=\frac{(\text { Wet weight }- \text { Dry weight })}{\text { Wet weight }} \times 100$

Moreover, burn wound was enforced HE staining.

Statistical analysis: Statistical evaluations were performed with the one-way Analysis of Variance (ANOVA) by SPSS (Version 15). Statistical significance was set at a $\mathrm{p}<0.05$. The results are presented as mean values \pm Standard Deviation (SD).

\section{RESULTS AND DISCUSSION}

MAP and core temperature: Large area cryotherapy made MAP and core body temperature decreased in rabbits, warm transfusion produced the opposite effects, no significant change between group D and A. At 2 and $24 \mathrm{~h}$ after scald, MAP in group B was significantly lower than group A but significant increased in group C. MAP of all groups had no significant difference at $48 \mathrm{~h}$ after scald (Fig. 1). At $2 \mathrm{~h}$ after scald: core body temperature of group B was significantly lower than group A but rose obviously in group C. At 24 and $48 \mathrm{~h}$ after scald: core body temperature was lower in group $\mathrm{B}$ and higher in group $\mathrm{C}$ compared with group $\mathrm{A}$ but had no statistical significant (Fig. 1).

Hemorheology: In this study, results indicated that large area cryotherapy had adverse effect on the stability of hemorheology. Rhs and EA in group B were significantly

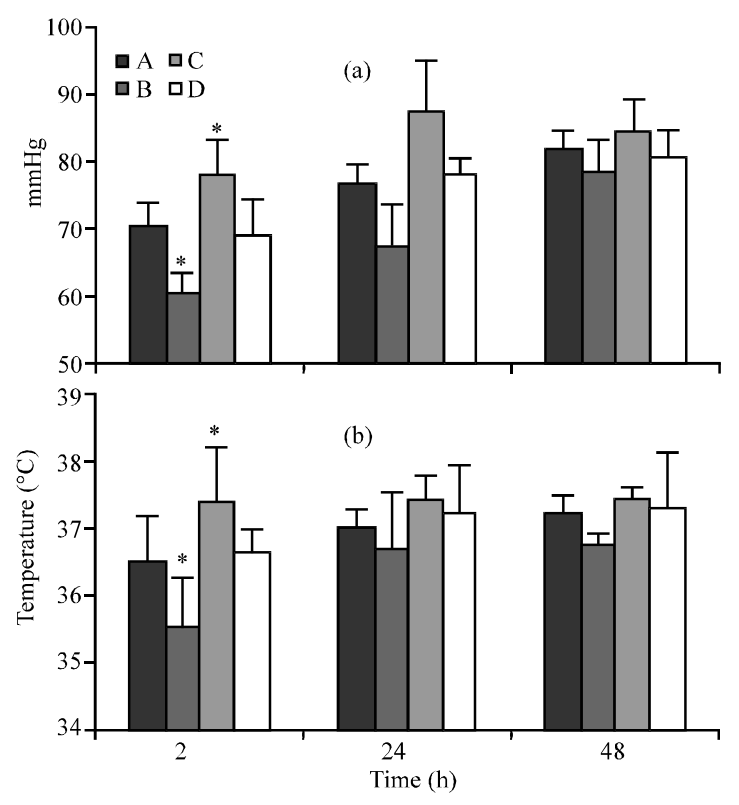

Fig. 1: a) MAP; b) Core body temperature in the 4 groups at 2, 24 and $48 \mathrm{~h}$ after burn; ${ }^{*} \mathrm{p}<0.05$ versus group A within time 
higher than group $\mathrm{A}$ at the 3 time points, Rls in group $\mathrm{B}$ was also higher only at 2 and $24 \mathrm{~h}$. Rls, Rhs and EA in group $\mathrm{C}$ were significantly lower than group $\mathrm{A}$ at $2 \mathrm{~h}$. There was no significant change of PV among groups and time points. Moreover, researchers found hemorheology parameters had no significant difference between group D and group A (Fig. 2).
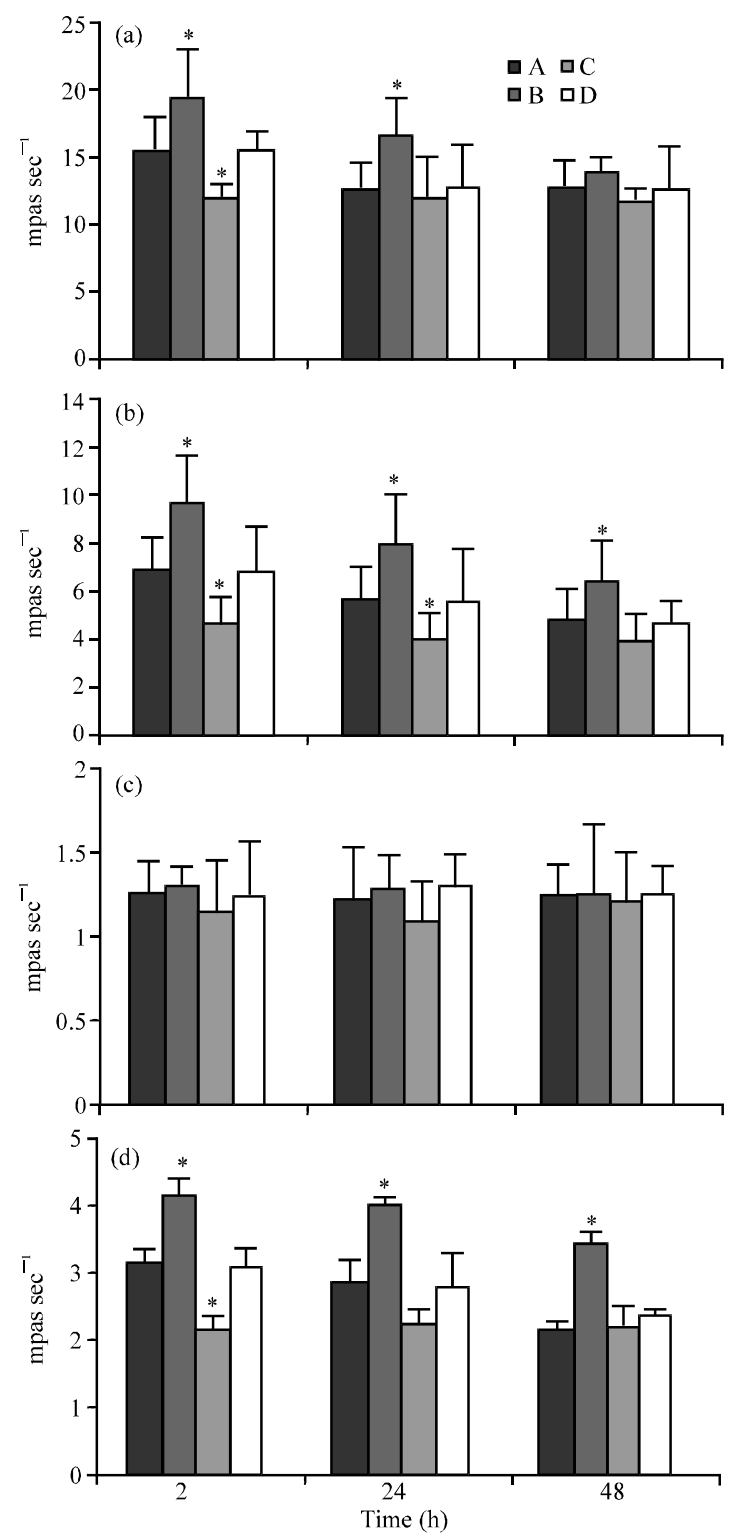

Fig. 2: Hemorheology indicators of: a) Rls; b) Rhs; c) PV and EA detected in the 4 groups at 2, 24 and $48 \mathrm{~h}$ after burn $\mathrm{p}<0.05$ vs. group A within time Rls: Reduced low shear viscosity; Rhs: Reduced high shear viscosity; PV: Plasma Viscosity; EA: Erythrocyte Aggregation
MPO and Moisture content : Both cryotherapy and the application of cryotherapy with warm transfusion reduced the levels of burn wound MPO. MPO in burn wound of group B was lower than group A at $2 \mathrm{~h}$ and $24 \mathrm{~h}$ after scald. MPO in burn wound of group D was lower than group $\mathrm{A}$ at all the time points but there was no obvious difference between group C versus group A (Fig. 3).

The application of cryotherapy combined with warm transfusion decreased moisture content of lung and burn wound. Moisture content of lung in group C and D was lower than group $\mathrm{A}$, moisture content of burn wound in group B and D was lower than group A (Fig. 3).

Inflammatory mediators: Overall, large area cryotherapy increased the concentration of inflammatory mediators in blood but the application of cryotherapy and warm transfusion decreased those significantly. $\mathrm{TNF} \alpha, \mathbb{L}-1 \beta$ and IL-6 in group B were increased versus that of group $A$ in early period after scald. In group $C$ and $D$, the inflammatory mediators were reduced significantly (Fig. 4).

Histological examination: Researchers examined the depth of burn wound at $48 \mathrm{~h}$ after scald by $\mathrm{HE}$ staining, results shown a similar depth between group $\mathrm{C}$ and $\mathrm{A}$ but group B and D displayed less dermal edema, collagen fiber fracture and inflammatory cell infiltration than the other 2 groups (Fig. 5).
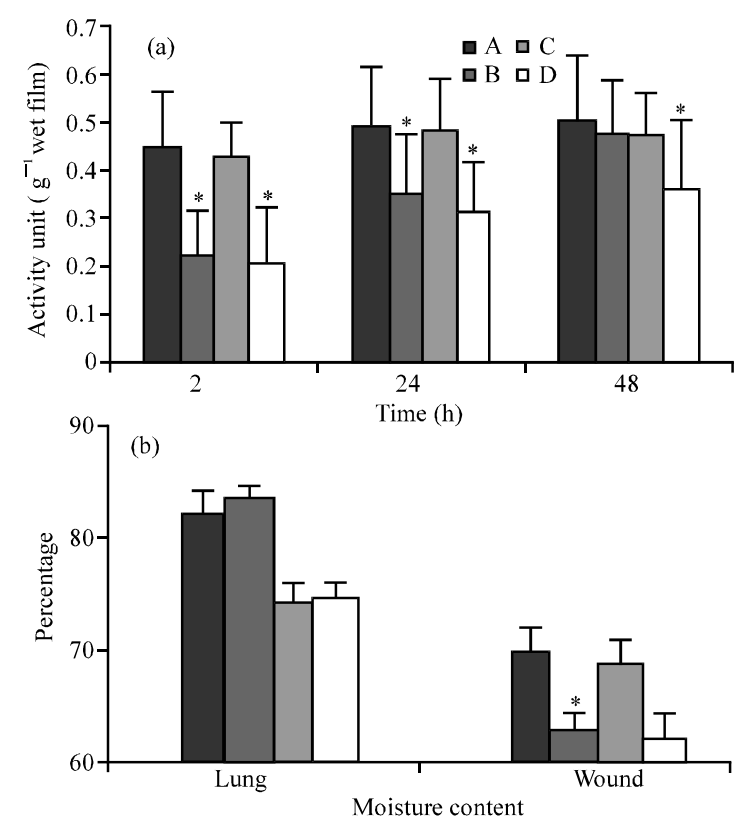

Fig. 3: a) Myeloperoxidase (MPO) in wound; b) Moisture content of lung and burn wound in the 4 groups $\mathrm{p}<0.05$ vs. group A within time 

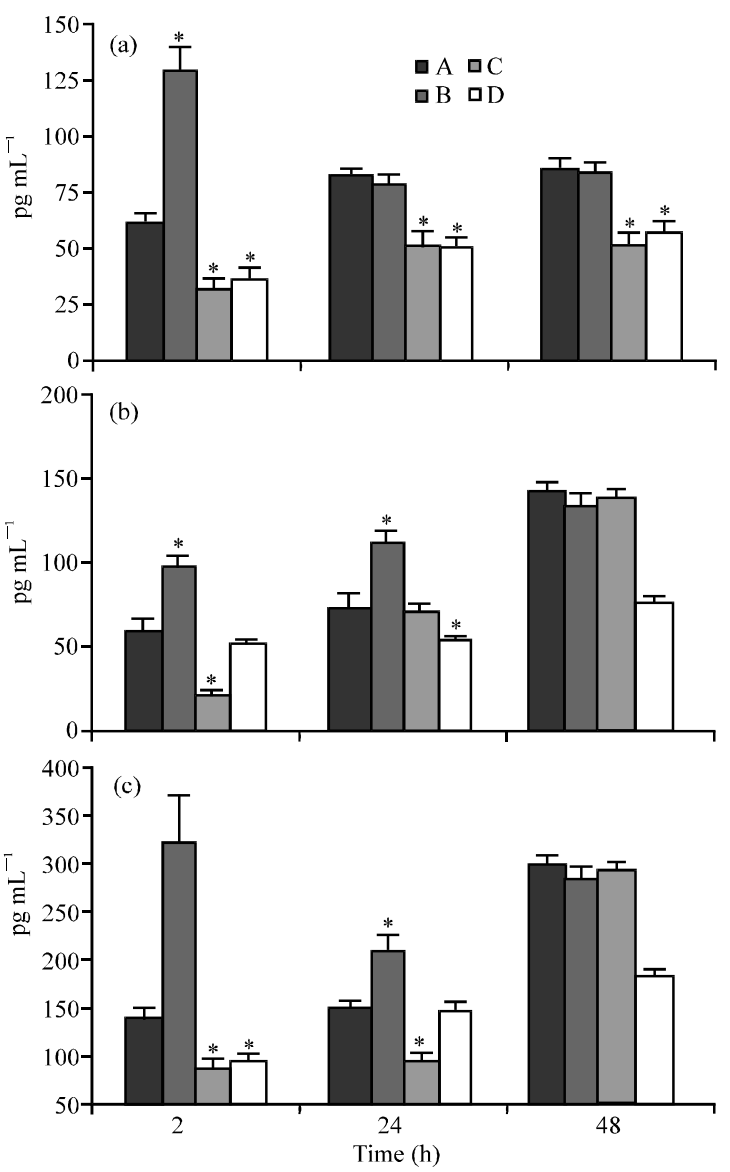

Fig. 4: Levels of; a: TNF- $\alpha$; b: IL-|B; c: IL-6 detected in the 4 groups at 2,24 and $48 \mathrm{~h}$ after burn $\mathrm{p}<0.05 \mathrm{vs}$. group A within time

In the field of burn therapeutic, cryotherapy as a simple and inexpensive method is attracting more and more attention in recent years. Now-a-days, the majority of ambulance services have utilized the technique of cooling to burn patients in the developed countries (Allison, 2002). Yang et al. (2009) identified the heat induced acute lung inflammation and electrolyte imbalance could be ameliorated by whole body cooling. Clinical application of cooling to burn wound treatment in first aid decreased complications, mortality and treatment costs significantly and accompanied the need for grafting reduction by $32 \%$ (Schlager and Iserson, 1988; Nguyen et al., 2002). Based on observation in vivo by fluorescence microscopy, researchers certified that cooling could obviously lighten the striated muscle inflammation stimulated by tumor necrosis factor and vasoconstriction disappeared soon after rewarming with no significant effect on local perfusion (Westermann et al., 1999; Thorlacius et al., 1998). In the research on cryotherapy indications, Lonnecker and
Schoder (2001) found no influence of cold-water treatment alone on the body temperature in small area of burn patients. Interestingly, Singer et al. (2010) reported that large burn patients with hypothermia wound significantly increase the mortality but prehospital cooling did not appear to contribute to hypothermia.

In the present study, researchers applied cryotherapy combined with warm transfusion to rabbits with full thickness scald covering $40 \%$ of TBSA then observed the vital signs, inflammatory mediators and hemorheology parameters in blood and moisture content of lung and burn wound. This data showen that cryotherapy decreased the core body temperature, MAP and wound MPO activity in rabbits and increased the levels of RSV/EA and inflammatory mediators compared to control group. Warm transfusion produced the opposite changes of the upper indicators. Lung moisture content in group $\mathrm{C}$ and wound moisture content in group $\mathrm{B}$ were lower compared to group A. Moreover, burn wound $\mathrm{MPO}$, moisture content of lung and wound and inflammatory mediators significantly descended in group D. Histological examination demonstrated that cryotherapy would reduce wound depth. These results indicates that warm transfusion can lighten the side effect of cryotherapy; the application of these two techniques is beneficial to treatment of burn wound and avoid the instability of life signs.

Many factors such as rate of body heat generation and heat loss, ambient temperature, tissue blood flow can change body temperature. Gentilello et al. (1990) estimated that $1 \mathrm{~L}$ of room temperature crystalloid solution decreased the mean body temperature by $0.25^{\circ} \mathrm{C}$. Oppositely, infusion of warm fluid is effective in keeping patients nearly normothermic and preventing postanesthetic shivering and it provide an easy and effective method for prevention of perioperative hypothermia (Hong-Xia et al., 2010). Considering the positive influence of warming treatment, researchers speculated that hypothermia caused by surface cooling might be improved by warming fluid treatment.

With the economic development, most hospitals in developing countries also have equipped with indoor temperature control system which is in favour of burn patients thermostasis. The experimental results further suggest that appropriate area cryotherapy combined with warm transfusion can be applied to large aera burn patients in early phase, especially the functional parts (such as hand) and exposed sites (such as face and neck). This method can alleviate burn depth of the important parts and meanwhile avoid hypothermia and hypotension caused by cryotherapy. Of course, researchers only observed the indicators within $48 \mathrm{~h}$ after scald. In the 

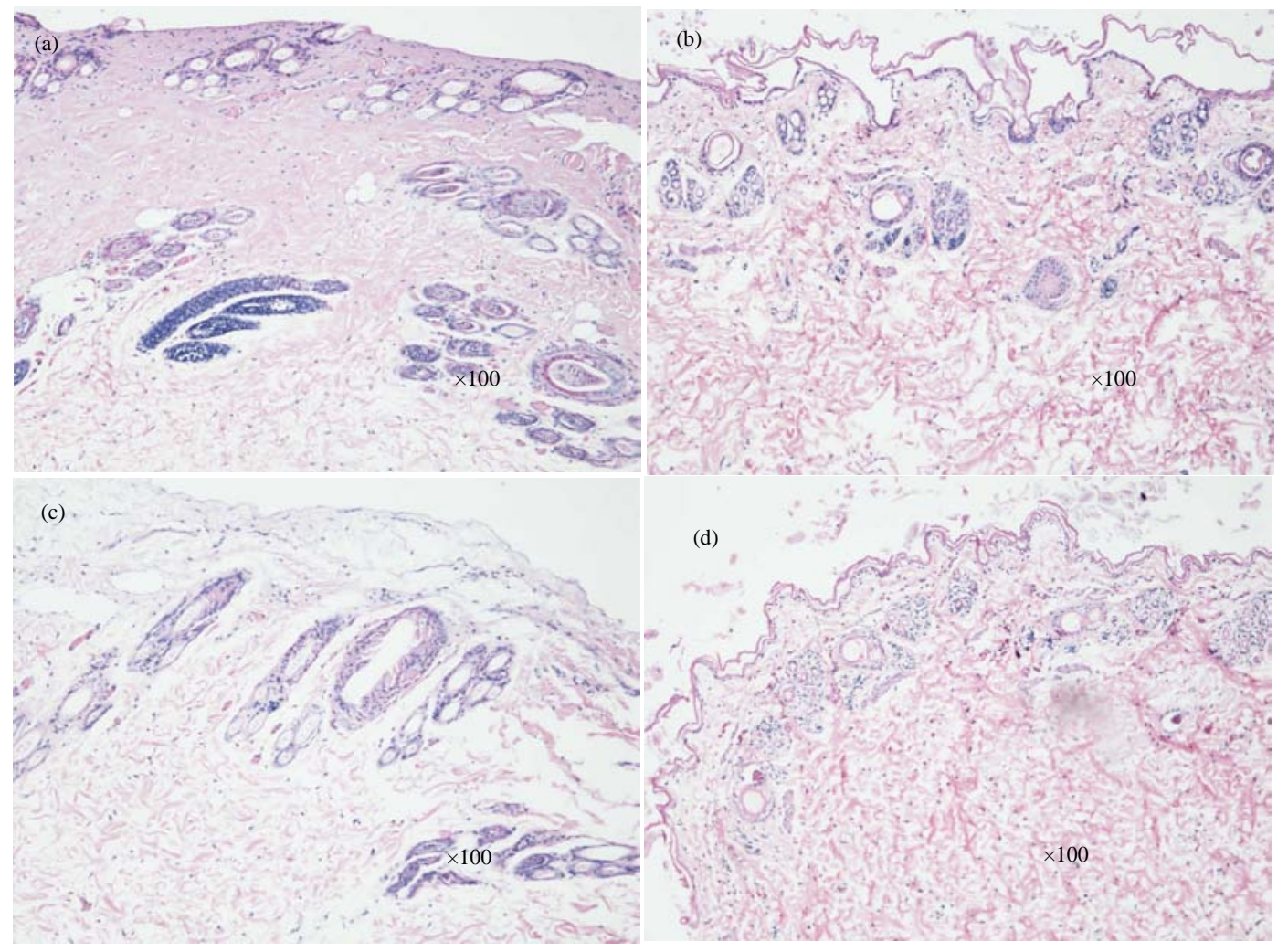

Fig. 5: HE staining of burn wound at $48 \mathrm{~h}$ after scald; Group A: Necrotic mass deep into the mid-dermis and numerous inflammatory cells infiltration and dilated vessels were seen; Group B: Superficial dermal collagen seems heat affected with moderate inflammation in the collagen and surrounding the hair follicles; Group C: A similar depth with group (A); Group D: The destruction of the tissue only reached into the up-dermis with minimal inflammatory changes in the dermis $(\mathrm{HE}, \times 100)$

subsequent research, researchers will prolong the observing time to verify the conclusion and further elucidate the influence of cryotherapy and warm transfusion on wound repair cells.

\section{CONCLUSION}

Histological examination demonstrated that cryotherapy would allievate wound depth. These results indicate that warm transfusion can prevent the side effect of cryotherapy, the application of these two techniques is beneficial to treatment of burn wound and avoid the instability of life signs.

\section{ACKNOWLEDGEMENTS}

This study was supported by grants from Gensci Burn Surgical Medical Scientific Research Fund and Graduate Innovation Program of Central South University. The researchers declare that they have no competing interests.

\section{REFERENCES}

Allison, K., 2002. The UK pre-hospital management of burn patients: Current practice and the need for a standard approach. Burns, 28: 135-142.

Gentilello, L.M., V. Cortes, S. Moujaes, M. Viamonte, T.L. Malinin, C.H. Ho and G.A. Gomez, 1990. Continuous arteriovenous rewarming: Experimental results and thermodynamic model simulation of treatment for hypothermia. J. Trauma, 30: 1436-1449.

Hasankhani, H., E. Mohammadi, F. Moazzami, M. Mokhtari and M.M. Naghgizadh, 2007. The effects of intravenous fluids temperature on perioperative hemodynamic situation, post-operative shivering, and recovery in orthopaedic surgery. Can. Oper. Room Nurs. J., 25: 20-24.

Hirase, Y., 1993. Postoperative cooling enhances composite graft survival in nasal-alar and ?ngertip reconstruction. Br. J. Plast Surg., 46: 707-711. 
Hong-Xia, X., Y. Zhi-Jian, Z. Hong and L. Zhiging, 2010. Prevention of hypothermia by Infusion of warm fluid during abdominal surgery. J. PeriAnesthesia Nurs., 25: $366-370$.

Horowitz, P.E., M.A. Delagarza, J.J. Pulaski and R.A. Smith, 2004. Flow rates and warming efficacy with hotline and ranger blood/fluid warmers. Anesth Analg, 99: 788-792.

Jackie, H. and R. Sukh, 2004. First aid and treatment of minor burns. BMJ., 328: 1487-1489.

Jandera, V., D.A. Hudson, P.M. Wet, P.M. Innes and $\mathrm{H}$. Rode, 2000. Cooling the burn wound: Evaluation of different modalites. Burns, 26: 265-270.

Kurz, A., D.I. Sessler and R. Lenhardt, 1996. Perioperative normothermia to reduce the incidence of surgical wound infection and shorten hospitalization. N. Engl. J. Med., 334: 1209-1215.

Li, C., D. Yu and M.S. Li, 1997. Clinical and experimen tstudy of cooling therapy on burned wound. Zhonghua Yi Xue Za Zhi., 77: 586-588.

Lonnecker, S. and V. Schoder, 2001. Hypothermia in patients with burn injuries: Influence of pre-hospital treatment. Chirurg, 72: 164-167.

Meusers, P.J., J. Lautenschlager, M. Herrmann and R. Borst, 1972. [Animal experimental studies of burn disease. Effect of local cooling following scalding]. Res. Exp. Med., 159: 114-123.

Nakazawa, H. and M. Nozaki, 2005. Usefulness of warm fluid in acute burn resuscitation: An experimental study in dogs. Tohoku J. Exp. Med., 207: 149-155.

Nguyen, N.L., R.T. Gun, A.L. Sparnon and P. Ryan, 2002. The importance of immediate cooling-a case series of childhood burns in vietnam. Burns, 28: 173-176.
Ofeigsson, O.J., R. Mitchell and R.S. Patrick, 1972. Observations on the cold water treatment of cutaneous burns. J. Pathol., 108: 145-150.

Sawada, Y., S. Urushidate, T. Yotsuyanagi and K. Ishita, 1997. Is prolonged and excessive cooling of a scalded wound effective. Burns, 23: 55-58.

Schlager, D. and K.V. Iserson, 1988. Emergency burn treatment in Denmark. J. Emerg. Med., 6: 517-519.

Shafi, S., A.C. Elliot andL. Gentilello, 2005. Is hypothermia simply a marker of shock and injury severity or an independent risk factor for mortality in trauma patients? Analysis of a large national trauma registry. J. Trauma, 59: 1081-1085.

Singer, A.J., B.R. Taira, H.C. Thode, J.E. McCormack, M. Shapiro, A. Aydin and C. Lee, 2010. The association between hypothermia, prehospital cooling, and mortality in burn victims. Socie. Acad. Emergen. Med., 17: 456-459.

Thorlacius, H., B. Vollmar, S. Westermann, L. Torkvist and M.D. Menger, 1998. Effects of local cooling on microvascular hemodynamics and leukocyte adhesion in the striated muscle of hamsters. J. Trauma, 45: 715-719.

Westermann, S., B. Vollmar, H. Thorlacius and M.D. Menger, 1999. Surface cooling inhibits tumor necrosis factor-alpha-induced microvascular perfusion failure, leukocyte adhesion and apoptosis in the striated muscle. Surgery, 126: 881-889.

Yang, H.H., C.P. Chang, J.T. Cheng and M.T. Lin, 2009. Attenuation of acute lung inflammation and injury by whole body cooling in a rat heatstroke model. J. Biomed. Biotechnol., Vol. 2009. 10.1155/2009/768086. 